\title{
Implementation of infection prevention and control in acute care hospitals in Mainland China - a systematic review
}

\author{
Jiancong Wang ${ }^{1,2,3}$, Fangfei Liu ${ }^{4}$, Jamie Bee Xian Tan ${ }^{1,5}$, Stephan Harbarth ${ }^{1}$, Didier Pittet ${ }^{1}$ and Walter Zingg ${ }^{1,6^{*}}$
}

\begin{abstract}
Background: Healthcare-associated infections (HAls) and antimicrobial resistance (AMR) affect patients in acute-care hospitals worldwide. No systematic review has been published on adoption and implementation of the infection prevention and control (IPC) key components. The objective of this systematic review was to assess adoption and implementation of the three areas issued by the "National Health Commission of the People's Republic of China" in acute-care hospitals in Mainland China, and to compare the findings with the key and core components on effective IPC, issued by the European Centre for Disease Prevention and Control (ECDC) and the World Health Organization (WHO).

Methods: We searched PubMed and the Chinese National Knowledge Infrastructure for reports on the areas "structure, organisation and management of IPC", "education and training in IPC", and "surveillance of outcome and process indicators in IPC" in acute-care facilities in Mainland China, published between January 2012 and October 2017. Results were stratified into primary care hospitals and secondary/tertiary care hospitals.

Results: A total of 6580 publications were retrieved, of which 56 were eligible for final analysis. Most of them were survey reports ( $n=27)$, followed by observational studies $(n=17)$, and interventional studies $(n=12)$, either on hand hygiene promotion and best practice interventions $(n=7)$, or by applying education and training programmes $(n=5)$. More elements on IPC were reported by secondary/tertiary care hospitals than by primary care hospitals. Gaps were identified in the lack of detailing on organisation and management of IPC, education and training activities, and targets of surveillance such as central line-associated bloodstream infections, ventilator associated pneumonia, catheterassociated urinary tract infections, and Clostridium difficile infections. Information was available on adoption and implementation of 7 out of the 10 ECDC key components, and 7 out of the 8 WHO core components.

Conclusion: To variable degrees, there is evidence on implementation of all NHCPRC areas and of most of the ECDC key components and the WHO core components in acute care hospitals in Mainland China. The results are encouraging, but gaps in effective IPC were identified that may be used to guide future national policy-making in Mainland China.
\end{abstract}

Keywords: Healthcare-associated infection, Infection prevention and control, Hospital management, Systematic review, China, Adoption, Implementation

\footnotetext{
* Correspondence: walter.zingg@hcuge.ch

These results were presented in part as a poster presentation at the 7 th Geneva Health Forum, Precision Global Health in the Digital Age, Geneva, Switzerland, on April 102018

'Infection Control Program and WHO Collaborating Centre on Patient Safety, University of Geneva Hospitals and Faculty of Medicine, Rue Gabrielle Perret-Gentil 4, 1211 Geneva 14, Switzerland

${ }^{6}$ National Institute for Health Research Health Protection Research Unit in Healthcare Associated Infections and Antimicrobial Resistance, Imperial College of London, London, UK

Full list of author information is available at the end of the article
}

(c) The Author(s). 2019 Open Access This article is distributed under the terms of the Creative Commons Attribution 4.0 International License (http://creativecommons.org/licenses/by/4.0/), which permits unrestricted use, distribution, and reproduction in any medium, provided you give appropriate credit to the original author(s) and the source, provide a link to the Creative Commons license, and indicate if changes were made. The Creative Commons Public Domain Dedication waiver (http://creativecommons.org/publicdomain/zero/1.0/) applies to the data made available in this article, unless otherwise stated. 


\section{Introduction}

The prevention of healthcare-associated infections (HAIs) is a first priority for patient safety in acute-care hospitals worldwide [1-5]. Adherence to the key and core components of infection prevention and control (IPC) issued by the European Centre for Disease Prevention and Control (ECDC)-funded "Systematic Review and Evidence-based Guidance on Organisation of Hospital Infection Control" (SIGHT) group and the World Health Organization (WHO), respectively, contributes to prevent HAI and the spread of antimicrobial resistance $[6,7]$. The United Nations Sustainable Development Goals highlighted the importance of IPC as a contributor to safe and effective high-quality health service delivery [7]. Furthermore, WHO intends to support countries in the development of their own national IPC programmes [7].

The Asia-Pacific region has been described as a geographic source for emerging infectious diseases, including multidrug-resistant organisms and pathogens with pandemic potential [8]. The People's Republic of China is the largest economic body in the region and faces similar global health challenges towards HAI and emerging antimicrobial resistance, as other countries in the region $[2,3$, 8]. Little is known on how hospitals prevent HAIs and control the spread of multidrug-resistant microorganisms in Mainland China; in particular, there is lack of information on the availability and the implementation of the ECDC key components for effective IPC.

In 2006, the National Health Commission of the People's Republic of China (NHCPRC) published the "Nosocomial Infection Management Methods" (Decree No. 48), which are guidelines defining elements on the organisation of IPC at hospital level [9]. In 2018, hospital accreditation was linked to the NHCPRC elements by the "Accreditation regulation of control and prevention of healthcareassociated infection in hospitals" (WS/T 592-2018) [10]. The NHCPRC decree embraces three broad areas of IPC: 1) structure, organization and management of IPC;2) education and training in IPC; and 3) outcome and process indicator surveillance in IPC.

The aim of this systematic review was to assess adoption and implementation of elements of the three NHCPRC areas by acute care hospitals in Mainland China, and to compare the findings with the ECDC key components and the WHO core components in IPC.

\section{Methods}

\section{Search strategy}

This systematic review followed the "Preferred Reporting Items for Systematic Review and Meta Analysis" (PRISMA) guidelines [11]. We searched PubMed, the "Chinese National Knowledge Infrastructure" database, and the Cochrane library for any relevant document. In addition, we looked for guidelines on the official websites of the NHCPRC and the regional Ministries of Health in Mainland China.

Primary outcomes were: reporting on adopting, implementing (having) or analysing elements of the three NHCPRC areas. Secondary outcomes were: reporting on change of indicators (e.g. HAI or hand hygiene) by applying IPC practices. The search terms addressed the three IPC areas specified by the NHCPRC for acute care hospitals: 1) structure, organization and management of IPC; 2) education and training of IPC; and 3) surveillance of process and outcome indicators relevant to IPC. Search terms and key words for PubMed and the "Chinese National Knowledge Infrastructure" are summarized in Additional file 1: Tables S1A and S1B.

\section{Inclusion/exclusion criteria}

Any article was eligible for inclusion when all of the following criteria were met: 1) use of a quantitative, qualitative or combined (mixed-methods) method; 2) reporting on one of the primary and/or secondary outcomes; 3) publication between January 2012 and October 2017; and 4) publication either in English or Chinese. Articles were excluded if they met one of the following criteria: 1) conference papers, editorials, or letters; 2) duplicated results; 3) risk factor analysis without information on the use of any IPC practice; 4) non-acute healthcare setting; or 5) outbreak investigations.

\section{Data extraction}

Title, abstract and full text review were performed by two individual researchers (JW, FL). Disagreements were resolved by consensus, and, when necessary, discussed with a third researcher (WZ). Data extraction was stratified by two hospital categories (primary care and secondary/tertiary care hospitals). Definitions on hospital categories are provided in Additional file 1: Table S2. Articles were further categorised as survey reports, observational studies or interventional studies. The following data were extracted from survey reports: title, authors, publication year, province, total number of hospitals, and the number of hospitals applying specific elements of the three NHCPRC areas. The following data were extracted from observational studies: title, author, publication year, province, study aim, setting, surveillance protocol, sample size, study duration, methodology, and outcome. The following data were extracted from interventional studies: title, authors, publication year, province, study aim, population, intervention, comparison, study design and outcome. Data extraction for interventional studies followed the "PICO" (population - intervention - comparison - outcome) concept [11]. Data were verified by cross-checking (JW, FL and JBXT). Survey reports and observational studies were quality assessed by using the "Strengthening the Reporting of 
Observational Studies in Epidemiology" (STROBE) checklist (Additional file 1: Tables S3A and S3B) [12]. Interventional studies were quality assessed by using the "Integrated quality Criteria for the Review Of Multiple Study designs" (ICROMS) checklist (Additional file 1: Tables S3C and S3D) [13]. Findings were stratified by the three NHCPRC areas, and compared with the ECDC key components [6], and the WHO core components [7].

\section{Statistical analysis}

Frequencies of elements mentioned in the survey reports were calculated on hospital level (with the corresponding $95 \%$ confidence interval), and stratified by hospital category. The difference of each identified element between hospital categories was tested by Pearson's ChiSquare test. Statistical analysis was performed using STATA version 14.0 (Stata Corporation, College Station, Texas, USA). Results of observational and interventional studies were summarized descriptively.

\section{Results}

From a total of 6580 titles and abstracts, 56 articles were eligible for data extraction and analysis (Fig. 1): 27 survey reports on structure, organisation and management of IPC (Table 1); 17 observational studies (8 single and 9 multicentre studies) measuring outcome and process indicators (Table 2); 5 interventional studies (5 single centre studies) applying education and training (Table 3); and 7 interventional studies (6 single- and 1 multicentre centre studies) testing the effectiveness of IPC strategies, mostly applying a multimodal strategy $(n=5)$ (Table 4$)$.

\section{NHCPRC area "structure, organisation and management of IPC"}

The search terms addressing the NHCPRC area on "structure, organisation and management of IPC" identified 27 survey reports summarizing the results of 1634 hospitals: 8 (29.6\%) reports on 440 primary care hospitals, $17(63.0 \%)$ reports on 1127 secondary/tertiary care hospitals, and $2(7.4 \%)$ reports on 26 primary- and 41 secondary/tertiary care hospitals combined (Table 1). The results of this area were divided into six elements (Table 1). Quality was moderate and low in eight and two of the 10 survey reports from primary care hospitals, respectively (Additional file 1: Table S4A). Quality was high, moderate and low in 1,14 , and 4 of the 19 survey reports from secondary/tertiary care hospitals, respectively (Additional file 1: Table S4B). Table 1, Additional file 1: Table S4A and Table S4B summarize the details on the reported elements, stratified by hospital types.

\section{Structure, organisation and management, guideline provision} Most primary care hospitals had an IPC committee (71.1\%), a formal IPC programme (61.9\%), and provided IPC guidelines (57.7\%). Most secondary/tertiary care hospitals had an IPC committee (98.1\%), performed feedback on IPC indicators (93.6\%), and provided IPC guidelines (85.8\%). No information on feedback, allocated

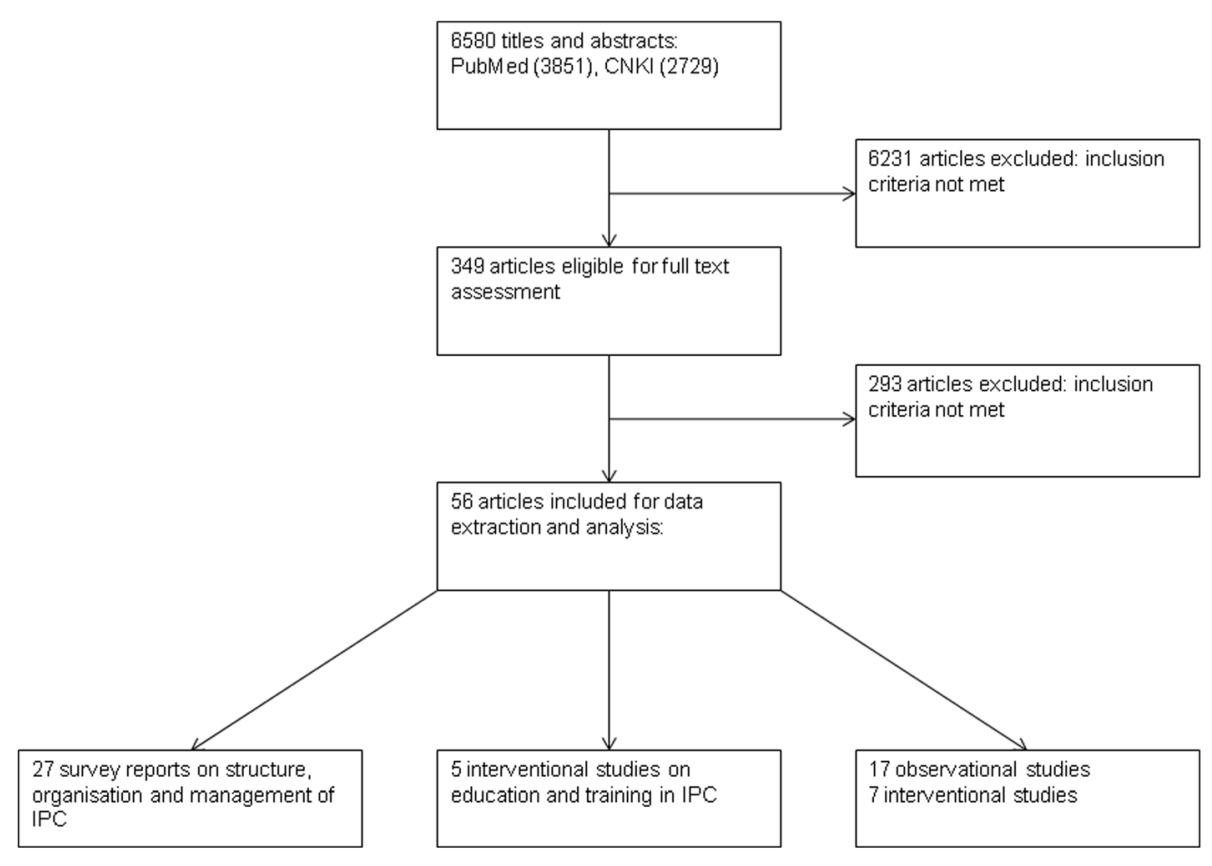

CNKI: China National Knowledge Infrastructure; IPC: infection prevention and control

Fig. 1 Systematic review profile - Systematic review on infection prevention and control in Mainland China, 2012-2017 


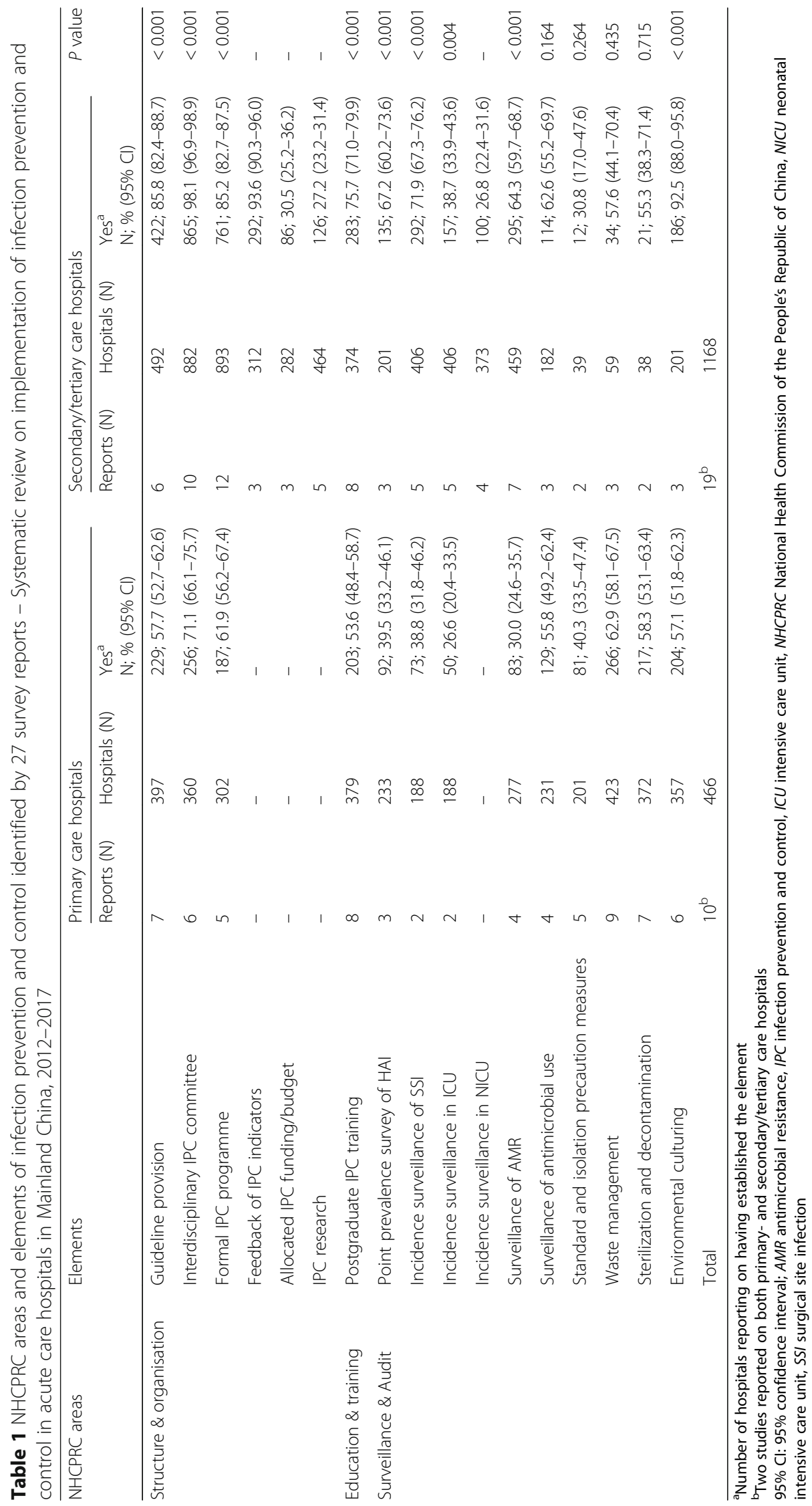




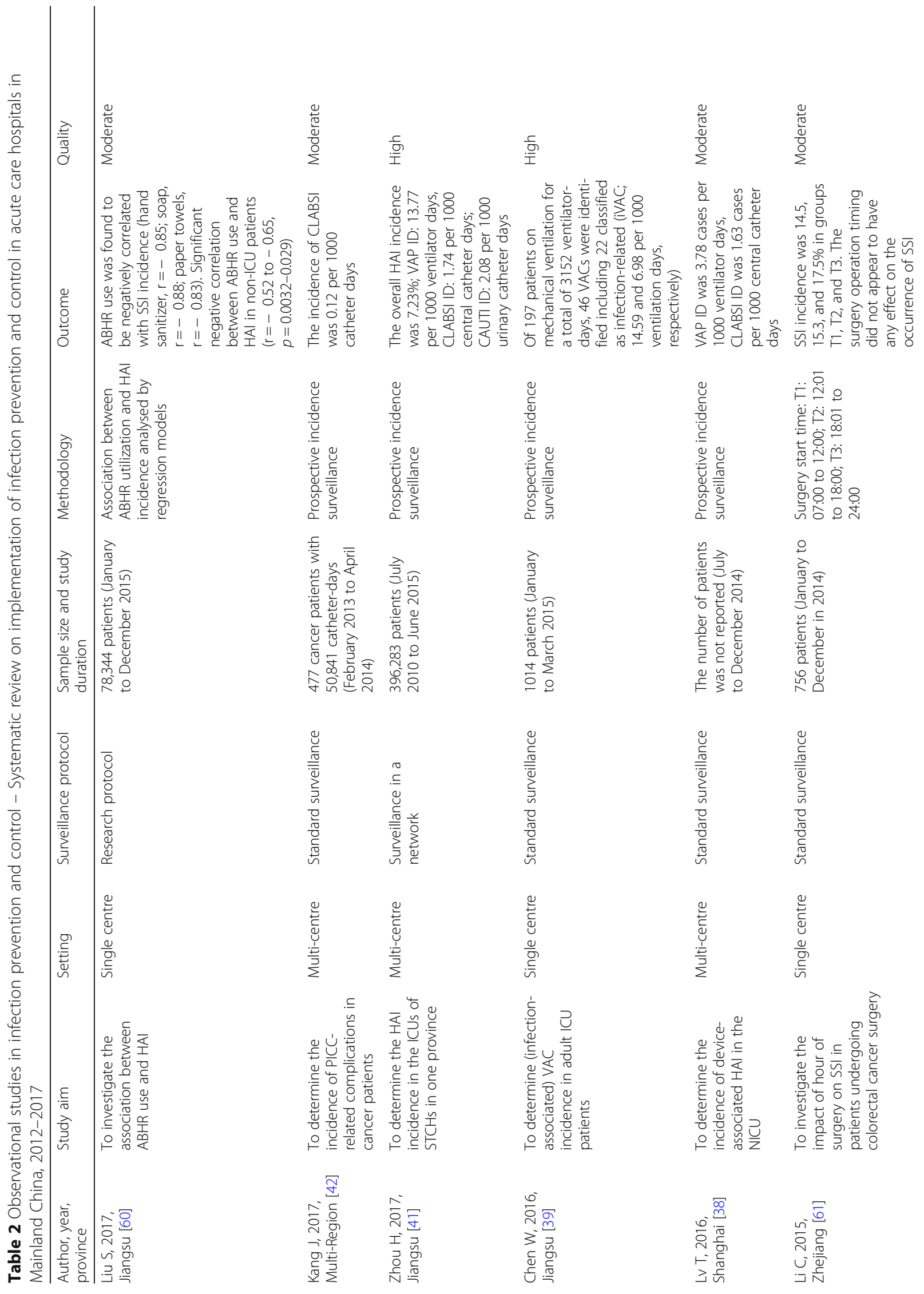




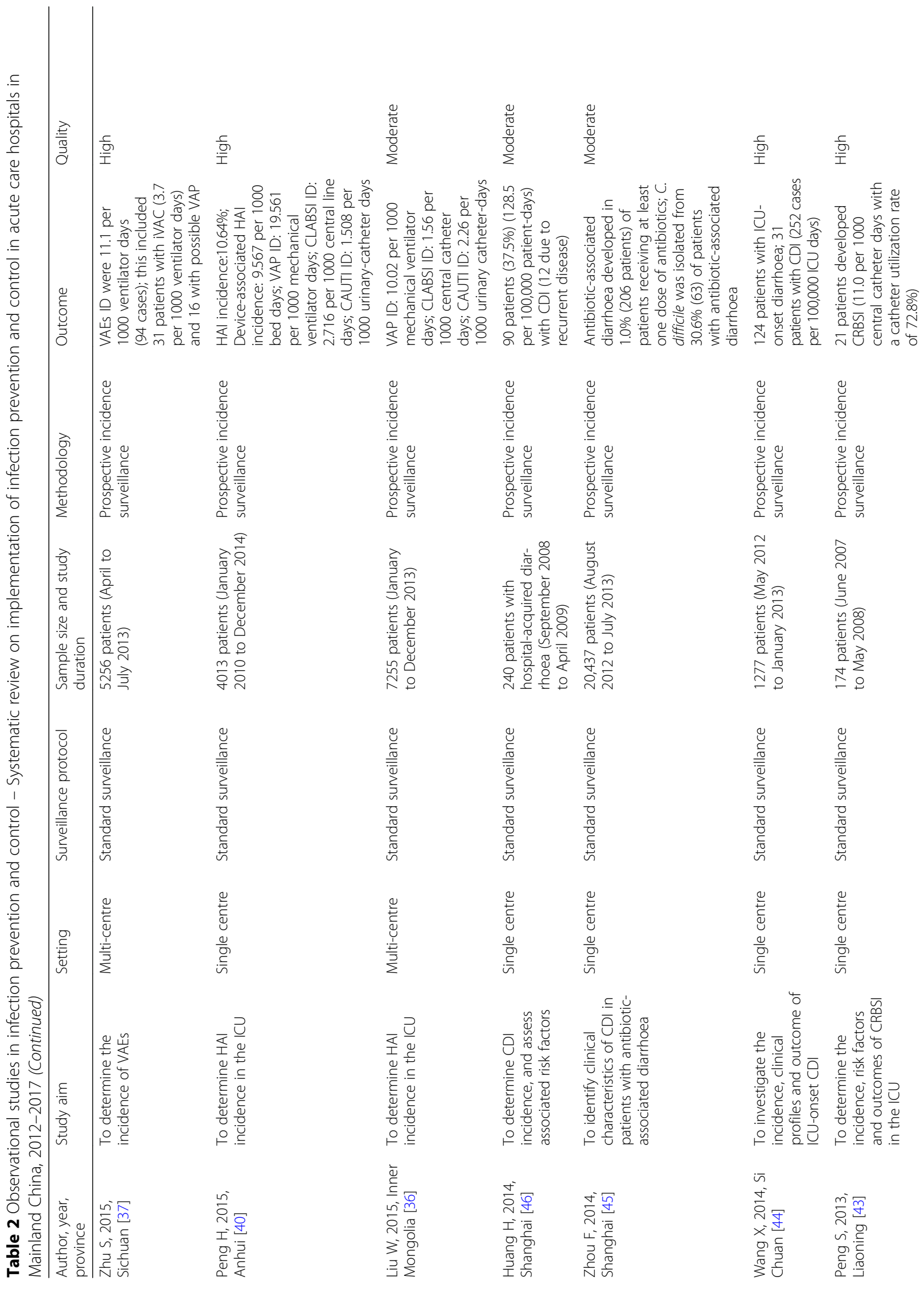




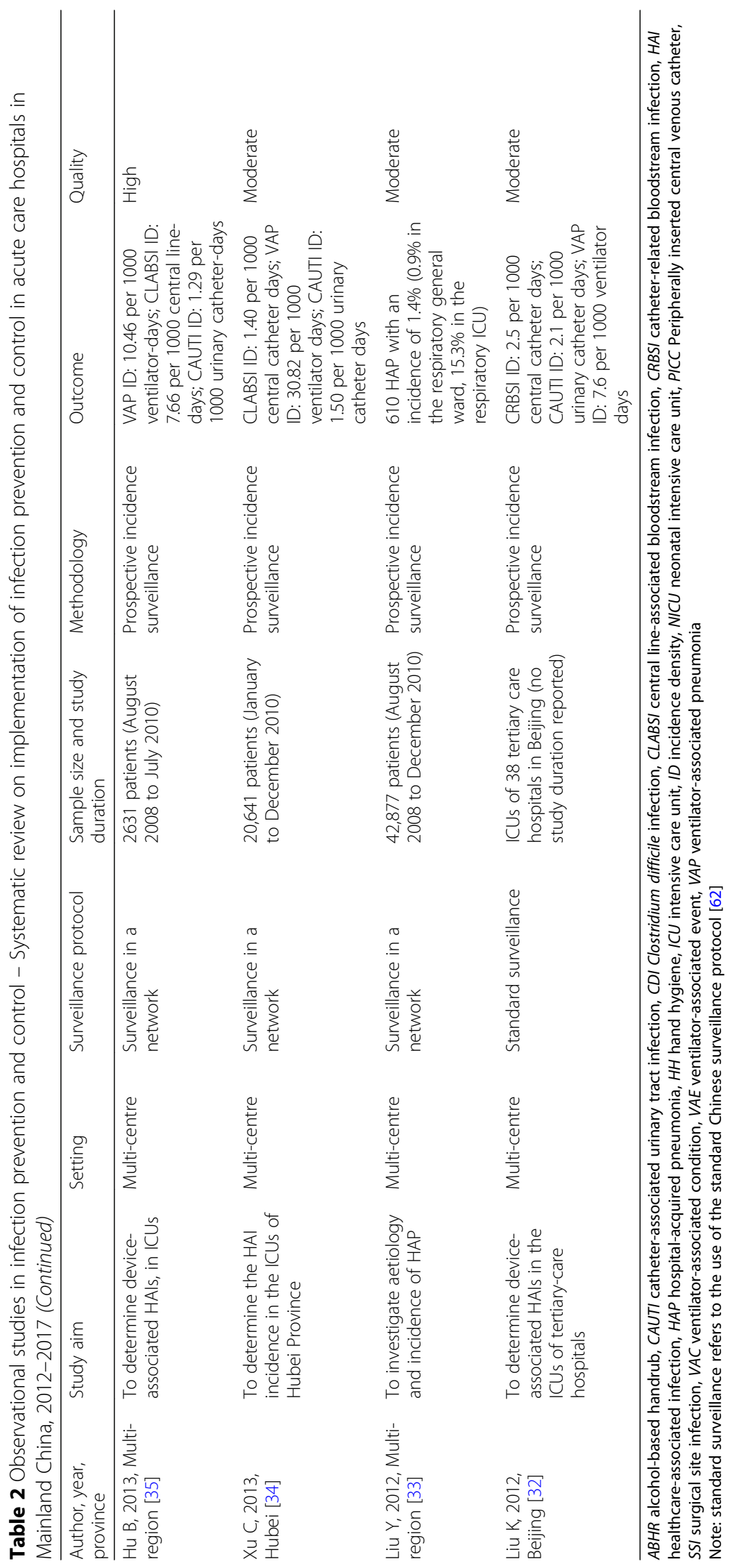




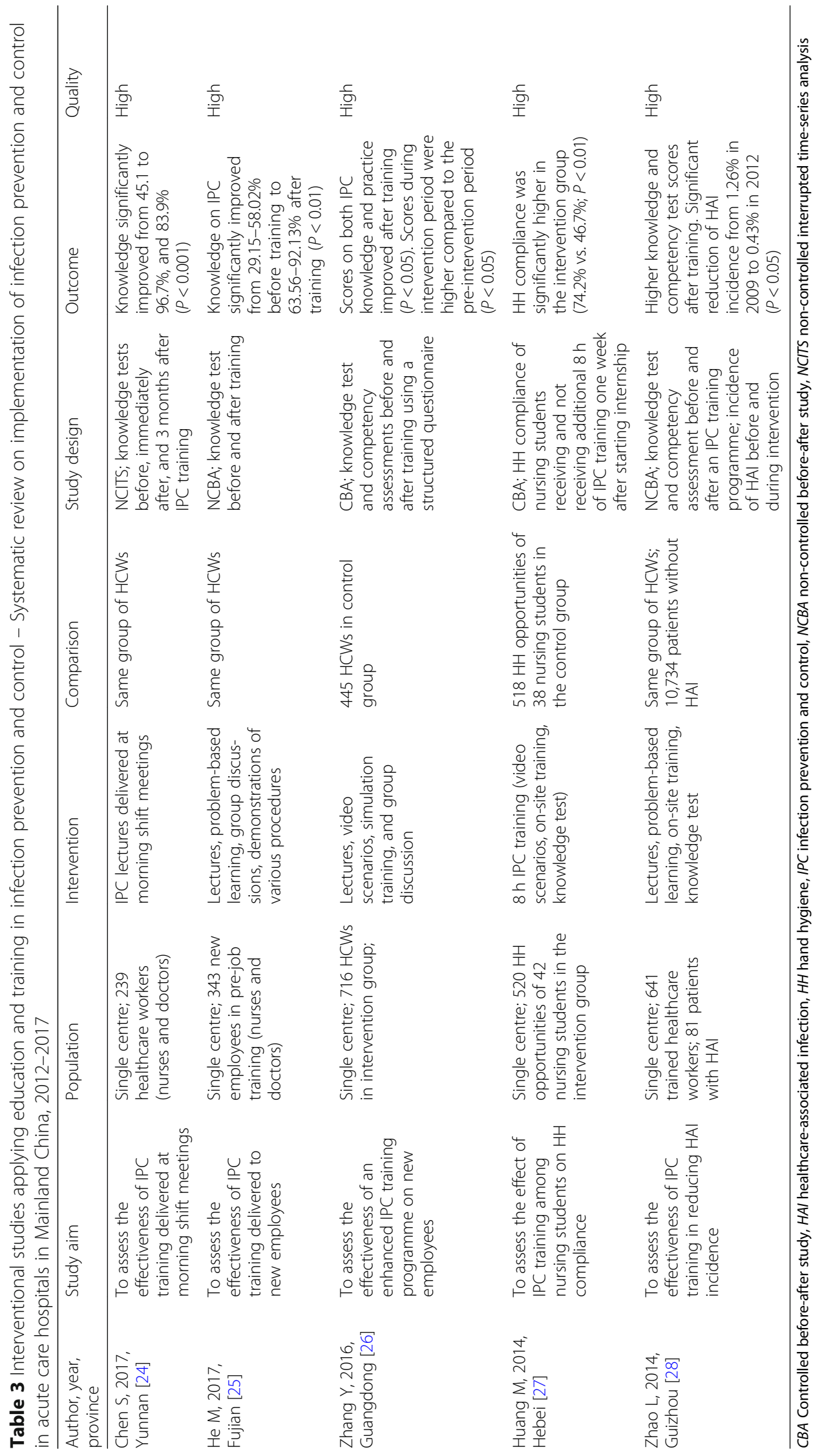




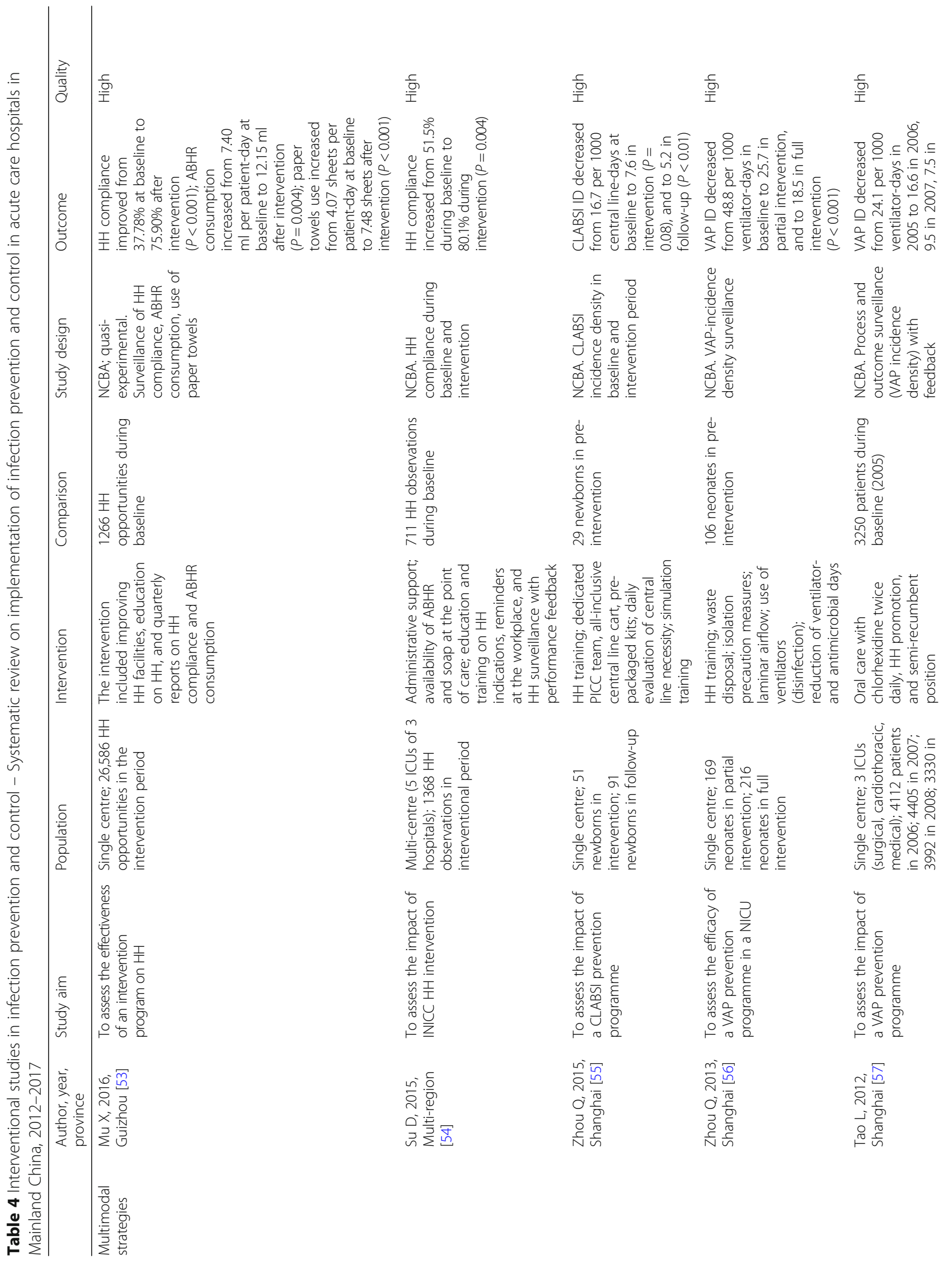




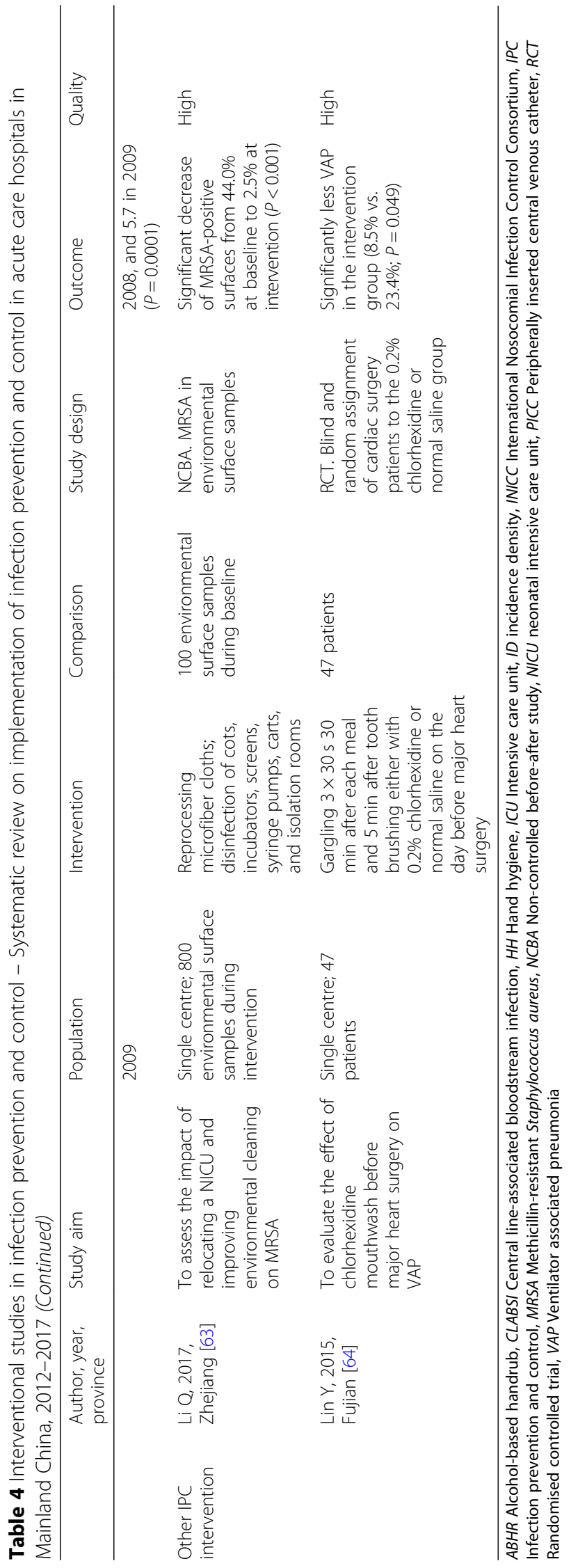


IPC funding/budget, and IPC research was identified for primary care hospitals. The frequencies of the elements were significantly different between hospital types, in favour for secondary/tertiary care hospitals. Only secondary/tertiary care hospitals reported numbers on IPC staff. The pooled ratios of IPC professionals, IPC doctors and IPC nurses were $0.51(0.48-0.53), 0.13(0.11-0.14)$, and $0.31(0.28-0.33)$ per 100 beds, respectively.

\section{Education and training in IPC}

Significantly more secondary/tertiary care hospitals offered regular, postgraduate IPC training compared to primary care hospitals (Table 1 ). However, the survey reports did not describe details on target population, training content, or frequency of training activities.

\section{Indicator and outcome surveillance in IPC}

The results of this area were stratified by "surveillance" and "auditing" and divided into 10 elements (Table 1). Surveillance of antimicrobial use (55.8\%) was the most reported element in primary care hospitals, followed by HAI point prevalence surveys (39.5\%), and incidence surveillance of surgical site infection (SSI) (38.8\%) (Table 1). No information was available on HAI incidence surveillance in neonatal intensive care units. The most frequently audited NHCPRC element in primary care hospitals was waste management (62.9\%), followed by sterilization and medical device decontamination (58.3\%), and environmental culturing (57.1\%) (Table 1). Incidence surveillance of SSI (71.9\%) was the most reported surveillance element in secondary/tertiary care hospitals, followed by point prevalence surveys (67.2\%), and surveillance of antimicrobial resistance (64.3\%) (Table 1). The most frequently audited NHCPRC element in secondary/tertiary care hospitals was environmental culturing $(92.5 \%)$, followed by waste management (57.6\%), and sterilization and medical device decontamination (55.3\%) (Table 1).

\section{NHCPRC area "Education and training in IPC"}

In addition to the above-mentioned survey reports, the search terms addressing the NHCPRC area on "Education and training in IPC" identified 5 single centre interventional studies: two non-controlled before-after studies, two controlled before-after studies, and one non-controlled interrupted time-series study. The quality of all five interventional studies was high (Additional file 1: Table S3C). Table 3 summarizes the details of the studies. Education and training in IPC was delivered via lectures, problem-based learning, (focus) group discussion, video scenarios, and simulation training. Two studies targeted new staff, whereas one focused on nursing students. Training activities were associated with improvement of IPC knowledge, increase of hand hygiene compliance, and reduction of HAIs.

NHCPRC area "outcome and process indicator surveillance" The search terms addressing the NHCPRC area on "outcome and process indicator surveillance" identified 17 observational studies (Table 2) and 7 interventional studies (Table 4). Of the 17 observational studies, 7 and 10 were of high and moderate quality, respectively (Table 2). All seven interventional studies were of high quality (Additional file 1: Table S3C). Five of the interventional studies applied a multimodal strategy, and measured either outcome indicators $(n=3)$ or process indicators $(n=2)$ (Table 4).

\section{Observational studies on outcome- and process indicator surveillance}

Table 2 summarizes the findings of the 17 observational studies: five measured device-associated HAIs, three allcause HAIs, three Clostridium difficile infections (CDI), two ventilator-associated pneumonias (VAPs), two central line-associated bloodstream infections (CLABSIs), one SSIs, and one hospital acquired pneumonia. Twelve studies applied the standard Chinese surveillance protocol, four applied a network protocol other than the official document, and one applied a research protocol.

\section{Interventional studies on outcome and process indicator surveillance}

Table 4 summarizes the details of the 7 interventional studies: six non-controlled before-after studies and one randomized controlled trial. Due to variation in intervention and outcome measurement, no meta-analysis was performed. Five studies used a multimodal strategy addressing hand hygiene improvement, CLABSI prevention, and VAP prevention. One study successfully tested pre-operative chlorhexidine mouthwash on VAP reduction, whereas another study reported MRSA reduction in the environment by improved cleaning practices.

\section{Mapping to key/core components in IPC}

This systematic review found information on all three NHCPRC areas, which are directly linked to the three ECDC key components on structure and organisation of IPC programmes, education and training in IPC, and performing surveillance (in a network) with timely feedback $[6,7]$. However, many of the survey reports also reported on elements linked to other ECDC key components such as provision and appropriate promotion of (locally adapted) guidelines, and performing audits. Furthermore, some of the interventional studies improved the provision of alcohol-based handrub at the point of care, or used new catheter insertion kits and trolleys, 
which are elements linked to the "materials and ergonomics" ECDC key component. Most of the interventional studies applied a multimodal strategy. Together, this systematic review identified information on 7 of the 10 ECDC key components, and 7 of the 8 WHO core components, respectively (Table 5).

\section{Discussion}

To our best knowledge, this is the first systematic review summarizing adoption and implementation of IPC in acute care hospitals in Mainland China. This review fills a research gap on the adoption and implementation of IPC in Mainland China (Table 6), highlighting, which of the key/core components recommended by ECDC/ WHO have been adopted and implemented, and which need further attention. It also offers an overview on the distribution of strategies and elements available in primary care hospitals compared to secondary/tertiary care hospitals. The observational and interventional studies complete the findings of the survey reports, offering a more granular picture on IPC activities in acute care hospitals in Mainland China. To various degrees, there is evidence that seven of the ECDC key components have been adopted and implemented in acute care hospitals in Mainland China.

\section{Structure, organisation and management of infection prevention and control}

Effective IPC in an acute care hospital needs an IPC programme with sufficient staffing and an allocated budget, support from the hospital management, and well defined duties and targets. The official structure requirements for IPC in Chinese hospitals (Additional file 1: Figure S1) are often not met. Only two-thirds of primary care hospitals have an IPC programme and an IPC committee. No information was found for any of the other elements of this NHCPRC area. The majority of secondary/tertiary care hospitals have an IPC programme, but only a third has an allocated budget. It is difficult to estimate the challenges on the proper functioning of IPC, but it has been shown that competing resources may have a negative impact on effective IPC [14]. Staffing, as identified in several reports is at minimal level $[6,7,15]$, comparable to other surveys $[16,17]$. However, high staff turnover, particularly among IPC doctors [18-20], is even of more concern than understaffing. This is partially explained by low salaries and limited career tracks [19]. Only 4.4\% of IPC doctors were satisfied with their position in one survey [19]. They were assigned to that position by hospital management, often against their will [19]. As a result, most IPC departments are managed by junior IPC doctors and IPC nurses. Due to the hierarchical gap between doctors and nurses, as well as between junior and senior doctors, IPC professionals face structural challenges, and struggle in influencing behaviour change [21].

Almost all secondary/tertiary care hospitals have an IPC committee in place. However, the importance of IPC is not always recognized by hospital management, and IPC committee members do not always participate actively in the committees $[19,20]$. Most secondary/tertiary care hospitals indicated to have a feedback mechanism in place. This is positive given the various IPC activities in surveillance and auditing. However, no details are available about format, target population, and frequency of feedbacks. Too often, information is conveyed to the hospital management only, and data reach healthcare workers too late, if at all, to be meaningful and impactful [22, 23].

A low proportion of primary care hospitals indicated to have IPC guidelines. This is surprising given that various national IPC documents are available $(n=30)$, and that implementation is mandatory for many of them $(n$ = 17) (Additional file 1: Table S5).

Table 5 Comparison with ECDC key components and WHO core components - Systematic review on implementation of infection prevention and control in acute care hospitals in Mainland China, 2012-2017

\begin{tabular}{lllll}
\hline & NHCPRC areas [9] & $\begin{array}{l}\text { Current systematic } \\
\text { review }\end{array}$ & $\begin{array}{l}\text { Core components } \\
\text { (WHO) [7] }\end{array}$ & $\begin{array}{l}\text { Key components } \\
\text { (ECDC) [6] }\end{array}$ \\
\hline Structure, organisation and management of IPC programmes & $\sqrt{ }$ & $\sqrt{ }$ & $\sqrt{ }$ & $\sqrt{ }$ \\
Provision and promotion of IPC guidelines & $\sqrt{ }$ & $\sqrt{ }$ & $\sqrt{ }$ & $\sqrt{ }$ \\
IPC education and training & $\sqrt{ }$ & $\sqrt{ }$ & $\sqrt{ }$ & $\sqrt{ }$ \\
Outcome and process indicator surveillance & $\sqrt{ }$ & $\sqrt{ }$ & $\sqrt{ }$ & $\sqrt{ }$ \\
Monitoring/auditing of IPC practices with individual feedback & $\sqrt{ }$ & $\sqrt{ }$ & $\sqrt{ }$ \\
Application of multimodal intervention strategies & N/A & $\sqrt{ }$ & $\sqrt{ }$ \\
Built environment, materials and equipment for IPC & N/A & N/A & $\sqrt{ }$ & $\sqrt{ }$ \\
Workload, staffing and bed occupancy & N/A & N/A & N/A \\
Engagement of champions & N/A & N/A & $\sqrt{ }$ \\
Positive organizational culture & N/A & N/A & $\sqrt{ }$
\end{tabular}

Note: N/A: Not available information after data searching; NHCPRC National Health Commission of the People's Republic of China 
Table 6 Gaps of the three NHCPRC focus areas - Systematic review on implementation of infection prevention and control in acute care hospitals in Mainland China, 2012-2017

1. Structure, organisation and management of infection prevention and control

- Limited IPC budget for IPC programmes;

- High IPC staff turnover, particularly among IPC doctors;

- Limited recognition by hospital management;

- Limited feedback of the results to healthcare professionals.

2. Education and training in infection prevention and control

- Limited resources for IPC education and training;

- Little experience with team-and task-oriented learning, or peer-to-peer

teaching education;

- Little experience with implementation strategies;

3. Surveillance of outcome and process indicators

- Few prospective incidence surveillance programmes

- Little antimicrobial stewardship programmes in primary-care hospitals;

- Little effort towards targeted MDRO screening of patients on admission

IPC infection prevention and control, NHCPRC National Health Commission of the People's Republic, MDRO multidrug-resistant microorganism

\section{Education and training in infection prevention and control}

Half of primary- and three-quarters of secondary/tertiary care hospitals indicated to have postgraduate IPC education and training in place. Education and training should be team- and task-oriented, frontline workers should take part in the preparation and execution process (ideally peerto-peer teaching), the content should follow local guidelines, and implementation should be multimodal [6]. Unfortunately, survey reports lack details on the target population, contents, delivery methods, and frequency. Thus, it is difficult to assess the available resources for education and training and whether they are adequate. The use of multimodal strategies was identified in five interventional studies that successfully reduced HAIs by improving IPC knowledge and increasing hand hygiene compliance [24-28]. Professionals working in IPC need knowledge and skills on management and implementation research [29]. With the "European Certificate for Infection Control", the European Society for Clinical Microbiology and Infectious Diseases has created a platform to offer comprehensive IPC training beyond hygiene to doctors $[29,30]$. Implementation research and management are not part of IPC training in Mainland China. The basic and intermediate skill levels focus on legal aspects, mandatory surveillance, definitions, diagnosis, HAI classification, HAI prevention, and hand hygiene (Additional file 1: Tables S6A and S6B) [31]. The contents of the advanced level were not sufficiently specified in the documents to allow conclusions on delivering skills regarding on the topic of project management and implementation research [31].

\section{Surveillance of outcome and process indicators Prevalence surveys, incidence surveillance of outcome- and process indicators}

A range of surveillance activities were identified in the survey reports and in the observational studies, with significant differences between the hospital types. Many hospitals perform regular prevalence surveys, but given that yearly prevalence surveys on HAI are mandatory in Mainland China, the proportion of primary- (40\%) and secondary/tertiary care hospitals (60\%) is surprisingly low. Most prospective incidence surveillance measures SSI, which is similar to a recent European survey [22].

Survey reports do not specify device-association (such as CLABSI; CAUTI; or VAP); and there is no prospective CDI surveillance. This is particularly interesting considering that the 17 observational studies reported on VAP or other ventilator-associated events $(n=10)$ [32-41], CLABSI $(n=9)$ [32, 34-36, 38, 40-43], CAUTI $(n=6)$ $[32,34-36,40,41]$, and CDI $(n=3)$ [44-46]. The absence of detailing device-associated HAI-types and specifying CDI in regular surveillance reports is of concern taking into account the fact that problems, particularly with CDI (13-25 CDI/10,000 patient-days) [44, 46], were identified by some of the observational studies.

Not even two thirds of the hospitals perform surveillance on antimicrobial resistance and antimicrobial use, although this is mandatory since 2011 (Additional file 1: Table S5) [47, 48]. This is of concern given the challenge of emerging resistance in the Asia Pacific region [1, 3]. In addition, no information was identified on antimicrobial stewardship, which, at least according to some reports, is not yet established in primary care hospitals $[3,49]$. In 2016, the CHINET surveillance programme reported $7.0 \%$ of Enterobacteriaceae being resistant to carbapenems, 38.4\% of Staphylococcus aureus being resistant to methicillin, and $45.2 \%$ of Enterobacteriaceae expressing extended-spectrum beta-lactamases [50]. These numbers are alarming, and IPC should be empowered both in recognition and resources to prevent cross-transmission of multidrug-resistant microorganisms in acute care hospitals.

\section{Monitoring/auditing}

Standard and isolation precaution measures, waste management, sterilization and medical device decontamination, and environmental cultures are audited. Interestingly, there is no difference in the frequencies between the hospital types except for environmental cultures. Almost all secondary/tertiary care hospitals perform routine environmental cultures (in intensive care, operating theatres, central sterile services departments, endoscopy suites, haemodialysis centres, and dentistry departments), which is of questionable value outside of outbreaks [51]. These resources could be better directed towards targeted screening of patients at risk of carrying multidrug-resistant microorganisms [52].

\section{Bundles and multimodal interventions}

Consistent with the ECDC key components [6], five of the seven interventional studies used a multimodal 
strategy to improve hand hygiene, and reduce CLABSI and VAP [53-57]. Two studies on hand hygiene combined leadership engagement, provision of alcohol-based handrub at the point of care, feedback, and reminders at the workplace $[53,54]$. One study on CLABSI prevention [55] and two studies on VAP prevention [56, 57] applied "bundles", and partially followed the recommendations from SHEA/IDSA $[58,59]$. These studies were performed in Eastern China, where the socioeconomic status is high, and therefore more resources might be available for implementing IPC measures and conducting studies (Additional file 1: Table S7) [3].

\section{Limitations}

This review has limitations. First, the data on the first NHCPRC area about IPC structure, organisation and management came from survey reports using questionnaires. This limits detailing, and the proportion of hospitals correctly implementing IPC elements may be overestimated. Second, the survey reports and studies in the final analysis originated mainly from regions with higher socioeconomic status, and thus, may not be representative for Mainland China as a whole. Third, publication bias may have occurred by the fact that we checked only scientific data sources and confined search on one English and one Chinese database. Fourth, there is no mandatory reporting system for HAI incidence in Mainland China. Studies on incidence surveillance are mainly retrospective. Thus, data on outcome indicators are limited. Fifth, methodological heterogeneity of the observational and interventional studies limited comparability; and thus, conducting a formal meta-analysis was not possible. However, the aim of this systematic review on describing adoption and implementation of elements of the three NHCPRC areas was still met. Sixth, the search terms of this systematic review were based on the three NHCPRC areas. We considered the concept of the ECDC key components too new to serve as a starting point for a search based on scientific literature. The search strategy based on recommendations by the Chinese healthcare authorities was a more pragmatic approach. However, the results still covered 7 of the 10 ECDC key components, and 7 of the $8 \mathrm{WHO}$ core components. The only lacking key components were about frontline staffing, integrating champions in the implementation of IPC strategies, and fostering a positive organisational culture.

\section{Conclusion}

To variable degrees, there is evidence on implementation of all NHCPRC areas and of most of the ECDC key components and the WHO core components in acute care hospitals in Mainland China. The results are encouraging, but gaps in effective IPC were identified that may be used to guide future national policy-making in Mainland China.

\section{Additional file}

\begin{abstract}
Additional file 1: Table S1A and S1B. Search terms for PubMed and the China National Knowledge Infrastructure (Chinese database). Table S2. Definition of hospital types. Table S3A and S3B. Strengthening the Reporting of Observational Studies in Epidemiology checklist for survey reports and observational studies. Table S3C and S3D. Integrated Quality Criteria for Review of Multiple Study Designs for interventional studies. Table S4A and S4B. Survey reports of primary care hospitals and secondary-/tertiary care hospitals. Table S5. Chinese national guidelines related to infection prevention and control, 2009-2018. Table S6A and S6B. Details of education and training programme on infection prevention and control in Mainland China. Table S7. Geographical distribution of survey reports, observational studies and interventional studies in the final analysis. Figure S1. Organisation and structure on infection prevention and control in Chinese hospitals (Three levels). (DOCX 180 kb)
\end{abstract}

\section{Abbreviations \\ CAUTI: Catheter-associated urinary tract infection; CDI: Clostridium difficile infection; CLABSI: Central line-associated bloodstream infection; ECDC: European Centre for Disease Prevention and Control; HAl: Healthcare-associated infection; IPC: Infection prevention and control; MRSA: Methicillin-resistant Staphylococcus aureus; NHCPRC: National Health Commission of the People's Republic of China; SIGHT: Systematic Review and Evidence-based Guidance on Organisation of Hos- pital Infection Control; SSI: Surgical site infection; VAP: Ventilator-associated pneumonia; WHO: World Health Organization}

\section{Acknowledgements \\ None.}

\section{Funding}

JW was supported and funded by Chinese Professional Talent Training Program, Dong Guan City, Guangdong Province, People's Republic of China.

\section{Availability of data and materials}

The datasets used and/or analysed during the current study are available from the corresponding author on reasonable request.

\section{Authors' contributions}

JW and WZ established the study protocol. JW and FL preformed literature search and data extraction. JW, FL and JBXT did data verification and crosschecking. Data analysis was done by JW and WZ. JW, JBXT and WZ wrote the first draft of the manuscript. All authors (JW, FL, JBXT, SH, DP, WZ) reviewed and contributed to subsequent drafts. All authors had full access to the study data and approved the final version.

Ethics approval and consent to participate

Not applicable

\section{Consent for publication}

Not applicable

Competing interests

The authors declare that they have no competing interests.

\section{Publisher's Note}

Springer Nature remains neutral with regard to jurisdictional claims in published maps and institutional affiliations.

\footnotetext{
Author details

${ }^{1}$ Infection Control Program and WHO Collaborating Centre on Patient Safety, University of Geneva Hospitals and Faculty of Medicine, Rue Gabrielle Perret-Gentil 4, 1211 Geneva 14, Switzerland. ' Institute of Global Health, Faculty of Medicine, University of Geneva, Geneva, Switzerland. ${ }^{3}$ Department of Infection Control, Dong Guan Hospital of Traditional Chinese Medicine, Dong Guan City, Guang Dong Province, China. ${ }^{4}$ Department of Nosocomial Infection Management, The Second Affiliated Hospital, Xi'an Jiaotong University, Xi'an, Shaanxi Province, China. ${ }^{5}$ Department of Microbiology, Singapore General Hospital, Singapore, Singapore. ${ }^{6}$ National Institute for
} 
Health Research Health Protection Research Unit in Healthcare Associated Infections and Antimicrobial Resistance, Imperial College of London, London, UK.

Received: 24 October 2018 Accepted: 30 January 2019 Published online: 11 February 2019

\section{References}

1. Allegranzi B, Bagheri Nejad S, Combescure C, Graafmans W, Attar H, Donaldson L, et al. Burden of endemic health-care-associated infection in developing countries: systematic review and meta-analysis. Lancet. 2011;377:228-41.

2. Wang J, Hu J, Harbarth S, Pittet D, Zhou M, Zingg W. Burden of healthcareassociated infections in China: results of the 2015 point prevalence survey in dong Guan City. J Hosp Infect. 2017;96:132-8.

3. Wang J, Liu F, Tartari E, Huang J, Harbarth S, Pittet D, et al. The prevalence of healthcare-associated infections in mainland China: a systematic review and Meta-analysis. Infect Control Hosp Epidemiol. 2018;39:701-9.

4. Burke JP. Infection control - a problem for patient safety. N Engl J Med. 2003;348:651-6.

5. Magill SS, Edwards JR, Bamberg W, Beldavs ZG, Dumyati G, Kainer MA, et al. Multistate point-prevalence survey of health care-associated infections. N Engl J Med. 2014;370:1198-208.

6. Zingg W, Holmes A, Dettenkofer M, Goetting T, Secci F, Clack L, et al. Hospital organisation, management, and structure for prevention of healthcare-associated infection: a systematic review and expert consensus. Lancet Infect Dis. 2015:15:212-24.

7. World Health Organization. Guidelines on core components of infection prevention and control programmes at the national and acute health care facility level. 2016. Available at: http://www.who.int/gpsc/core-components.pdf. Accessed 22 Oct 2018.

8. Apisarnthanarak A, Mundy LM, Tantawichien T, Leelarasamee A. Infection prevention and control in Asia: current evidence and future milestones. Clin Infect Dis. 2017:64:549-550.

9. Ministry of Health of People's Republic of China. Nosocomial Infection Management Method (Decree No.48) (in Chinese). 2006. Available at: http:// www.gov.cn/flfg/2006-07/25/content_344886.htm. Accessed 22 Oct 2018.

10. National Health Commission of the People's Republic of China. Accreditation regulation of control and prevention of healthcare-associated infection in hospital (WS/T 592-2018) (in Chinese). 2018. Available at: http://www.nhfpc. gov.cn/ewebeditor/uploadfile/2018/05/20180523110555724.pdf. Accessed 22 Oct 2018.

11. Moher D, Liberati A, Tetzlaff J, Altman DG. Preferred reporting items for systematic reviews and meta-analyses: the PRISMA statement. PLoS Med. 2009;6:e1000097.

12. von Elm E, Altman DG, Egger M, Pocock SJ, Gotzsche PC, Vandenbroucke JP. The strengthening the reporting of observational studies in epidemiology (STROBE) statement: guidelines for reporting observational studies. Lancet. 2007:370:1453-7.

13. Zingg W, Castro-Sanchez E, Secci FV, Edwards R, Drumright LN, Sevdalis N, et al. Innovative tools for quality assessment: integrated quality criteria for review of multiple study designs (ICROMS). Public Health. 2016;133:19-37.

14. Clack L, Zingg W, Saint S, Casillas A, Touveneau S, da Liberdade Jantarada $F$, et al. Implementing infection prevention practices across European hospitals: an in-depth qualitative assessment. BMJ Qual Saf. 2018;27:771-80

15. Haley RW, Culver DH, White JW, Morgan WM, Emori TG, Munn VP, et al. The efficacy of infection surveillance and control programs in preventing nosocomial infections in US hospitals. Am J Epidemiol. 1985;121:182-205.

16. European Center for Disease Prevention and Control. Point prevalence survey of healthcare associated infections and antimicrobial use in European acute care hospitals. Stockholm: ECDC; 2013.

17. Hansen S, Zingg W, Ahmad R, Kyratsis $Y$, Behnke M, Schwab F, et al. Organization of infection control in European hospitals. J Hosp Infect. 2015;91:338-45

18. Zhang $Y$, Zhang $H$, Jin $F$, Zhang J. Current status of full-time staff of nosocomial infections control in Gansu province (in Chinese). Chin J Nosocomiol. 2013;23:3448-9.

19. Zhang Z, Han M, Yang Z, Wei Q, Wang X, Wei Q, et al. Current situation of full-time healthcare-associated infection management staff in Xianyang City of Shaanxi Province (in Chinese). Chin J Infect Control. 2017;16:635-8.
20. Liu S, Li C, Li L, Hou T, Ding L, Liu W, et al. Development of healthcareassociated infection management organizations in China in the past 30 years (in Chinese). Chin J Infect Control. 2016;15:648-53.

21. Yuan CT, Dembry LM, Higa B, Fu M, Wang H, Bradley EH. Perceptions of hand hygiene practices in China. J Hosp Infect. 2009;71:157-62.

22. Hansen S, Schwab F, Zingg W, Gastmeier P. Process and outcome indicators for infection control and prevention in European acute care hospitals in 2011 to 2012 - results of the PROHIBIT study. Euro Surveill. 2018;23(21). https://doi.org/10.2807/1560-7917.ES.2018.23.21.1700513.

23. Ren N, Wen X, Fu C, Li L, Hou T, Ding L, et al. Development and changing trend in monitoring of healthcare-associated infection in China (in Chinese). Chin J Infect Control. 2016;15:642-7.

24. Chen S, Han G, Li L, Xiong X. Training at morning shift meeting can improve awareness rate of healthcare-associated infection knowledge among health care workers (in Chinese). Chin J Infect Control. 2017;16:858-61.

25. He $M$, Lin $X$, Zeng $H$, Fang $W$, Lin X. Effect of pre-job training about healthcare-associated infections at military hospitals (in Chinese). J Prev Med Chin PLA. 2017;35:28-30

26. Zhang Y, Zheng D, Tian B, Chen X, Zhang S, Xu J. The effectiveness of training in improving the infection prevention and control knowledge among new healthcare professionals (in Chinese). Shenzhen J Integrated Traditional Chin and Western Med. 2016;26:177-8.

27. Huang $M, X u J$, Zhao $Y$, Chen $Y$. Effect of IPC training conducted by infection prevention and control professionals on hand hygiene compliance among nursing interns (in Chinese). J Bethune Med Sci. 2014;12:608-9.

28. Zhao L, Yang R, Yuan $H$. Role of hospital infection knowledge training in improving aseptic techniques of medical workers (in Chinese). Chin J Nosocomiol. 2014;24:3082-4.

29. Zingg W, Mutters NT, Harbarth S, Friedrich AW. Education in infection control: a need for European certification. Clin Microbiol Infect. 2015:21:1052-6.

30. European Committee on Infection Control. EUCIC launches a European training programme in Infection Prevention and Control in healthcare settings. 2018. Available at: https://www.escmid.org/fileadmin/src/media/ PDFs/3Research_Projects/EUCIC/WEB_EUCIC_Groningen_2018.pdf. Accessed 22 Octobe 2018

31. Wu A, Huang X, Li L, Gong Y, Liu C, Wang L, et al. Guideline for professional training about managing of healthcare associated infections WS/T 525-2016 (in Chinese). Chin J Infect Control. 2017:16:94-7.

32. Liu K, Yuan X, Wu Y, Xue W. Study on targeted surveillance of nosocomial infections in ICU of tertiary care hospitals in Beijing (in Chinese). Chin J Nosocomial. 2012;22:248-50.

33. Liu YN, Cao B, Wang H, Chen LA, She DY, Zhao TM, et al. Adult hospital acquired pneumonia: a multicenter study on microbiology and clinical characteristics of patients from 9 Chinese cities (in Chinese). Chin J Tuberc Respir Dis. 2012;35:739-46.

34. Xu C, Xiong W. Analysis of the targeted surveillance of nosocomial infections in ICUs of 22 hospitals (in Chinese). Modern Prev Med. 2013: 40:3432-4.

35. Hu B, Tao L, Rosenthal VD, Liu K, Yun Y, Suo Y, et al. Device-associated infection rates, device use, length of stay, and mortality in intensive care units of 4 Chinese hospitals: international nosocomial control consortium findings. Am J Infect Control. 2013;41:301-6.

36. Liu W, Tian Y, Zheng Z. Target surveillance of nosocomial infections in ICU of 28 hospitals in Inner Mongolia autonomous region during 2013 (in Chinese) Chin J Disinfect. 2015:32:675-7.

37. Zhu S, Cai L, Ma C, Zeng H, Guo H, Mao X, et al. The clinical impact of ventilator-associated events: a prospective multi-center surveillance study. Infect Control Hosp Epidemiol. 2015;36:1388-95.

38. Lv T, Zhang Y, Liu L, Hu X, Zhang X, Li F, et al. Environmental hygiene and nosocomial infections in neonatal intensive care unit: a multi-center survey (in Chinese). J Nurs Sci. 2016;31:92-4.

39. Chen WS, Liu J, Liu H, Song YY, Chen HY, Wang R, et al. Prospective evaluation on ventilator-associated events: a cohort study from eight intensive care units (in Chinese). Chin J Epidemiol. 2016;37:1148-51.

40. Peng H, Tao XB, Li Y, Hu Q, Qian LH, Wu Q, et al. Health care-associated infections surveillance in an intensive care unit of a university hospital in China, 2010-2014: findings of international nosocomial infection control consortium. Am J Infect Control. 2015:43:e83-5.

41. Zhou H, Jiang Y, Li Y, Zheng W, Shen L. Consecutive 6-year targeted monitoring on healthcare-associated infection in intensive care units in 176 hospitals (in Chinese). Chin J Infect Control. 2017;16:810-5. 
42. Kang J, Chen W, Sun W, Ge R, Li H, Ma E, et al. Peripherally inserted centra catheter-related complications in cancer patients: a prospective study of over 50,000 catheter days. J Vasc Access. 2017;18:153-7.

43. Peng S, Lu Y. Clinical epidemiology of central venous catheter-related bloodstream infections in an intensive care unit in China. J Crit Care. 2013; 28:277-83.

44. Wang X, Cai L, Yu R, Huang W, Zong Z. ICU-onset Clostridium difficile infection in a university hospital in China: a prospective cohort study. PLoS One. 2014;9:e111735

45. Zhou FF, Wu S, Klena JD, Huang HH. Clinical characteristics of Clostridium difficile infection in hospitalized patients with antibioticassociated diarrhea in a university hospital in China. Eur J Clin Microbiol Infect Dis. 2014;33:1773-9.

46. Huang H, Wu S, Chen R, Xu S, Fang H, Weintraub A, et al. Risk factors of Clostridium difficile infections among patients in a university hospital in Shanghai, China. Anaerobe. 2014;30:65-9.

47. Xiao Y, Zhang J, Zheng B, Zhao L, Li S, Li L. Changes in Chinese policies to promote the rational use of antibiotics. PLoS Med. 2013;10: e1001556.

48. Ministry of Health of People's Republic of China. The management approach of clinical application of antibiotics use (in Chinese). Chin J Frontiers of Med Sci. 2013;5:9-14.

49. Li Y, Liu C, Liu X, Han Z, Hua C, Wang X, et al. Current status of healthcare-associated infection organization management systems in primary medical institutions in China (in Chinese). Chin J Infect Control. 2016;15:694-7.

50. Hu F, Guo $Y$, Zhu D, Wang F, Jiang $X, X u Y$, et al. CHINET surveillance of bacterial resistance across China: report of the results in 2016. Chin J Infect Chemother. 2017;17:481-91.

51. U.S. Department of Health and Human Services Centers for disease control and prevention. Guidelines for environmental infection control in healthcare facilities. 2003. Available at: https://www.cdc.gov/infectioncontrol/pdf/ guidelines/environmental-guidelines.pdf. Accessed 22 Oct 2018.

52. Coia JE, Leanord AT, Reilly J. Screening for meticillin resistant Staphylococcus aureus (MRSA): who, when, and how? BMJ. 2014;348:g1697.

53. Mu X, Xu Y, Yang T, Zhang J, Wang C, Liu W, et al. Improving hand hygiene compliance among healthcare workers: an intervention study in a Hospital in Guizhou Province, China. Braz J Infect Dis. 2016;20:413-8.

54. Su D, Hu B, Rosenthal VD, Li R, Hao C, Pan W, et al. Impact of the international nosocomial infection control consortium (INICC) multidimensional hand hygiene approach in five intensive care units in three cities of China. Public Health. 2015;129:979-88.

55. Zhou Q, Lee SK, Hu XJ, Jiang SY, Chen C, Wang CQ, et al. Successful reduction in central line-associated bloodstream infections in a Chinese neonatal intensive care unit. Am J Infect Control. 2015;43:275-9.

56. Zhou Q, Lee SK, Jiang SY, Chen C, Kamaluddeen M, Hu XJ, et al. Efficacy of an infection control program in reducing ventilator-associated pneumonia in a Chinese neonatal intensive care unit. Am J Infect Control. 2013;41: 1059-64.

57. Tao L, Hu B, Rosenthal VD, Zhang Y, Gao X, He L. Impact of a multidimensional approach on ventilator-associated pneumonia rates in a hospital of Shanghai: findings of the international nosocomial infection control consortium. J Crit Care. 2012;27:440-6.

58. Klompas M, Branson R, Eichenwald EC, Greene LR, Howell MD, Lee G, et al. Strategies to prevent ventilator-associated pneumonia in acute care hospitals: 2014 update. Infect Control Hosp Epidemiol. 2014;35:S133-54.

59. Marschall J, Mermel LA, Fakih M, Hadaway L, Kallen A, O'Grady NP, et al. Strategies to prevent central line-associated bloodstream infections in acute care hospitals: 2014 update. Infect Control Hosp Epidemiol. 2014;35: S89-107.

60. Liu S, Wang M, Wang G, Wu X, Guan W, Ren J. Microbial characteristics of nosocomial infections and their association with the utilization of hand hygiene products: a hospital-wide analysis of 78,344 cases. Surg Infect. 2017; 18:676-83.

61. Li CX, An XX, Zhao B, Wu SJ, Xie GH, Fang XM. Impact of operation timing on post-operative infections following colorectal cancer surgery. ANZ J Surg. 2016;86:294-8.

62. National Health Commission of the People's Republic of China. Standard for nosocomial infection surveillance (in Chinese). 2009. Available at: http:// www.nhfpc.gov.cn/cmsresources/mohyzs/cmsrsdocument/doc5842.pdf. Accessed 22 Oct 2018
63. Li QF, Xu H, Ni XP, Lin R, Jin H, Wei LY, et al. Impact of relocation and environmental cleaning on reducing the incidence of healthcare-associated infection in NICU. World J Pediatr. 2017;13:217-21.

64. Lin YJ, Xu L, Huang XZ, Jiang F, Li SL, Lin F, et al. Reduced occurrence of ventilator-associated pneumonia after cardiac surgery using preoperative 0 . $2 \%$ chlorhexidine oral rinse: results from a single-Centre single-blinded randomized trial. J Hosp Infect. 2015;91:362-6.

\section{Ready to submit your research? Choose BMC and benefit from:}

- fast, convenient online submission

- thorough peer review by experienced researchers in your field

- rapid publication on acceptance

- support for research data, including large and complex data types

- gold Open Access which fosters wider collaboration and increased citations

- maximum visibility for your research: over $100 \mathrm{M}$ website views per year

At BMC, research is always in progress.

Learn more biomedcentral.com/submissions 